\title{
Turning Tissues Transparent by Optical Phase Conjugation
}

\author{
Meng Cui, Emily McDowell, Changhuei Yang \\ Electrical Engineering and Bioengineering
}

California Institute of Technology, 1200 E California Blvd, MC 136-93, Pasadena CA91104

Elastic optical scattering in biological tissue typically dominates over absorption by an order of magnitude or more. Being the dominant light interaction process, scattering prevents tissue from being transparent. Although the spectral properties [1] and angular profiles [2] of scattered light can reveal useful physiological information about a given tissue sample, scattered light is generally regarded as poor in imaging information. This is due to the severe deterioration of the incident light field, caused by disordered amplitude and phase modulation of its wavefront as it propagates through the tissue. Yet, it is also known that elastic optical scattering is a deterministic and time reversible process. In other words, if we can record the phase and amplitude of the propagating scattered light field completely and reproduce a back propagating optical phase conjugate (OPC) field or a timereversed light field this field should be able to retrace its trajectory through the scattering medium and return the original input light field.

$$
\text { A year ago, we }
$$

demonstrated and reported that OPC can indeed be used to suppress tissue scattering in tissues [3]. Progressing from that point, we recently demonstrated that the turbidity suppression by optical phase conjugation (TSOPC) is remarkably robust. We were able to observe TSOPC reconstruction through a tissue of thickness $7 \mathrm{~mm}$ and wavelength of $532 \mathrm{~nm}$. At this thickness and wavelength, the average number of scattering events experienced by a photon exceeds 200. Yet, our results show that it is possible to force some of these photons to retrace their paths through the tissue. In addition, we found that this phenomenon is observable even when the total amount of scattered wavefront collected is only $0.2 \%$ of the total.

(a)
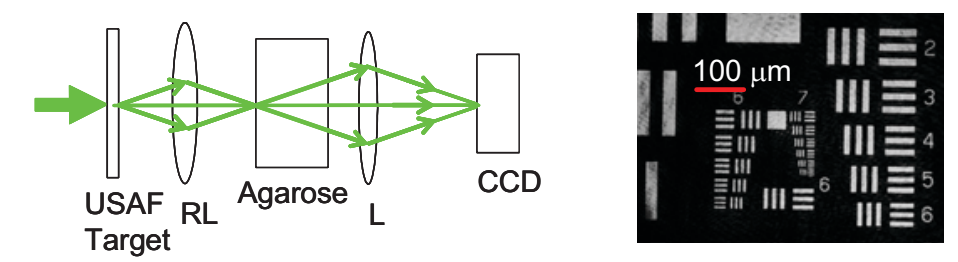

(b)
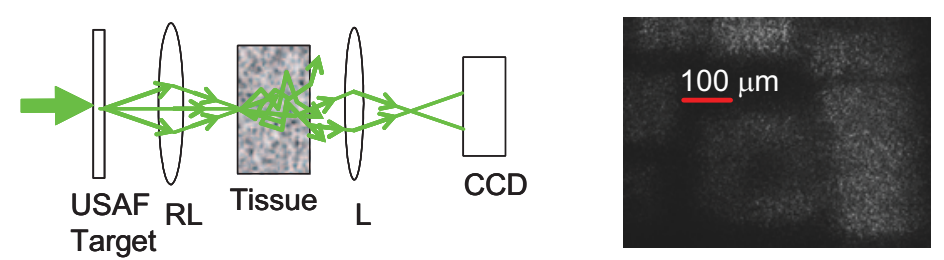

(c)
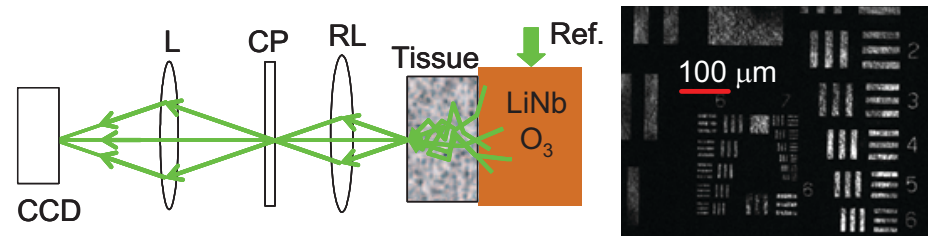

(d)
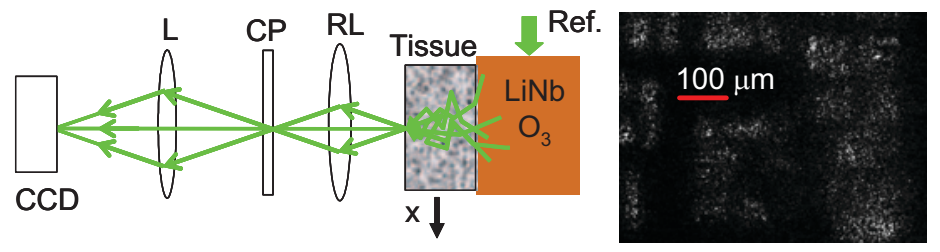

Figure 5: a) Transmission through agarose. b) Transmission through $0.5 \mathrm{~mm}$ thick chicken breast tissue. c) TS-OPC transmission. d) Degradation of TS-OPC transmission when sample is shifted. 
In a related study, we also found that the decay rate trend of this phenomenon in living tissue is on the orders of seconds. This finding is also counter-intuitive as one would expect cellular motions to rapidly disrupt the time-reversal condition over a much shorter time-scale.

We recently implemented a digital optical phase conjugation mirror by combining a spatial light modulator and a digital holography system. This system provides us with additional flexibility in modifying the scattered wavefront for TSOPC transmission experiments.

We will discuss these results in the presentation. We will also outline some of the applications that this phenomenon can be adapted to address.

[1] I. J. Bigio, S. G. Bown, G. Briggs, C. Kelley, S. Lakhani, D. Pickard, P. M. Ripley, I. G. Rose, and C. Saunders, "Diagnosis of breast cancer using elastic-scattering spectroscopy: preliminary clinical results," Journal of Biomedical Optics, vol. 5, pp. 221-228, Apr 2000.

[2] M. S. Sacks, D. B. Smith, and E. D. Hiester, "A small angle light scattering device for planar connective tissue microstructural analysis," Annals of Biomedical Engineering, vol. 25, pp. 678689, Jul-Aug 1997.

[3] Z. Yaqoob, D. Psaltis, M. S. Feld, and C. Yang, "Optical phase conjugation for turbidity suppression in biological samples " Nature Photonics, vol. 2, pp. 110 - 115, 2008. 